\title{
BMJ Open Adverse experiences of social adaptation in children with leukaemia: a qualitative study from China
}

\author{
Xinyue Tang (1) , ${ }^{1,2,3,4}$ Lin Mo, ${ }^{2,3,4,5}$ Qian Liu, ${ }^{1,2,3,4}$ Yixuan Liu, ${ }^{1,2,3,4}$ Lu Yu, ${ }^{2,3,4,6}$ \\ Yang Liu, ${ }^{2,3,4,7}$ Chunhai Gao ${ }^{8,9}$
}

To cite: Tang X, Mo L, Liu Q, et al. Adverse experiences of social adaptation in children with leukaemia: a qualitative study from China. BMJ Open 2022;12:e051953. doi:10.1136/ bmjopen-2021-051953

- Prepublication history for this paper is available online. To view these files, please visit the journal online (http://dx.do org/10.1136/bmjopen-2021051953).

Received 03 April 2021 Accepted 03 February 2022
Check for updates

\section{(C) Author(s) (or their} employer(s)) 2022. Re-use permitted under CC BY-NC. No commercial re-use. See rights and permissions. Published by BMJ.

For numbered affiliations see end of article.

Correspondence to

Lin Mo; molin999@126.com

\section{ABSTRACT}

Aim and objective This study aimed to build an indepth and comprehensive understanding of the social maladaptation experience of Chinese children with leukaemia of different ages.

Methods The purposive sampling method was adopted to conduct semistructured interviews with 20 Chinese children with leukaemia or their main caregivers and Colaizzi's method for qualitative research was used to organise and analyse the data. The study followed the guiding principles of the Standards for Reporting Qualitative Research.

Results The existing social adaptation problems of children with leukaemia of different ages were assessed. In terms of psychological maladjustment, children with leukaemia aged 2-7 years showed fear and excessive attachment, while children aged 8-18 years were characterised by impatience, irritability and anxiety. In terms of behavioural maladjustment, children aged 2-7 years presented less independent behaviour and dependence on electronics, while children aged 8-18 years showed learning obstruction and indulgence in mobile games.

Conclusions Targeted intervention strategies should be developed based on the physical and mental characteristics of children with leukaemia of different ages to improve existing social adaptation problems.

Relevance to clinical practice Early intervention for social adaptation of children with leukaemia should be considered. Children's social adaptation should be improved to prevent more adaptation problems so that they can return to their families and to the society without difficulty and improve their quality of life in the long term.

\section{INTRODUCTION}

In the process of interaction between individuals and their environment, social adaptation refers to people ultimately maintaining a dynamic balance with the social environment by adapting to it, adjusting themselves or changing the environment to maintain stability in physical and mental activities. ${ }^{1}$ Children with leukaemia are in a special period of growth and development. Two to threeyears of repeated hospitalisation, isolation from their peers and poor life experience

\section{Strengths and limitations of this study}

This is the first study to specifically describe the social adaptation experience of children with leukaemia of different ages and provides a useful reference for personalised care.

- The sample size was determined based on the principle of data saturation.

- The number of interviews was limited and there may be incomplete and inaccurate data on some topics.

- This study adopted consecutive sampling so there were certain limitations in the representativeness of the demographic characteristics of the selected children.

- The deficiencies in this study could be eliminated in future studies.

lead to various social adaptation problems in children with leukaemia due to changes in their living environment. ${ }^{2}$ This has led to the status quo of social maladjustment in children with leukaemia. A study found that the risk of maladjustment among childhood cancer survivors is as high as $23 \%$, with the highest risk among those with leukaemia. ${ }^{3}$ Children with leukaemia of different ages show various social maladaptations, which are mainly reflected in psychological and behavioural changes. In other words, both psychology and behaviour need to be adjusted. In China, the social adaptability of children with malignant tumours such as leukaemia is significantly worse than that of other children of the same age. ${ }^{4}$ Eventually, their studies, employment, marriage and family lives are affected. Therefore, it is urgent to improve the social adaptation of children with leukaemia. However, there is no indepth understanding of the social maladaptation experience of children with leukaemia. Children's life experiences can be intuitively understood through qualitative research. Tremolada et a $\bar{l}$ adopted semistructured interviews and a mixedmethod approach to examine children's and 
adolescents' narratives about their return to school after 2 years of treatment for leukaemia. Their aims were to collect their illness experiences and understand how they felt about school and their daily routines and to identify the best sociodemographic and illness predictors of good readaptation to school and daily life. Similarly, the present study used qualitative research methods to clarify the social maladaptation experience of children with leukaemia of different ages in China and identify their psychological and behavioural problems, with the goal of solving these problems in a targeted manner to improve the status quo of children's social maladaptation.

\section{Background}

In terms of incidence, leukaemia ranks first among paediatric malignancies and is a major disease that seriously threatens the lives and health of children. ${ }^{6}$ From 2016 to 2018, the average annual incidence of leukaemia among children aged 0-14 in China was 42.9 per million and the number of leukaemia cases every year was approximately $8000 .{ }^{7}$ Leukaemia might occur in children at any age, but the peak age is generally between birth and 14 years old. With significant progress in treatment methods, leukaemia and other blood system tumours have changed from a fatal to a chronic disease. ${ }^{8}$ Currently, the 5-year long-term survival rate of children with leukaemia is $87.8 \% .^{9}$ The burden of childhood cancer in China ranks second in the world, ${ }^{10}$ and childhood leukaemia survivors have the highest health burden among all childhood cancer survivors. Two-thirds of childhood leukaemia survivors have long-term health complications due to treatment, including psychosocial problems related to their illness experience. ${ }^{8}$ Some quantitative studies have identified short-term and long-term problems in the social adaptation of children with leukaemia, such as anxiety, depression and antisocial behaviour due to the disease itself and treatment. ${ }^{211} 12$ Once children with leukaemia reach adulthood, their risks of suicide, ${ }^{13}$ unemployment ${ }^{14}$ and sexual dysfunction ${ }^{15} 16$ become significantly higher than those of adults who have never had leukaemia. Early identification of social maladaptation in children with leukaemia and improving their social adaptation ability during hospitalisation can effectively prevent and control such social adaptation problems.

China attaches great importance to children with leukaemia. National Health Medical Development (2018) No. 16 declared that we must commit to their treatment, perfect the monitoring and evaluation intervention mechanism, and provide them with comprehensive and full-cycle health services. Social adaptation is an important indicator to evaluate the mental health characteristics of this group, and accurate assessment is key to guiding and evaluating the effect of social adaptation management in children with leukaemia. There have been quantitative studies on the overall quality of life or social adaptability of children with leukaemia. ${ }^{217}$ However, these studies were limited in reflecting the current status of social adaptation in children with leukaemia, as the assessment tools or methods they used were not specifically designed for this group. Moreover, social adaptation involves psychological changes; thus, qualitative research is more advantageous in truly reflecting the social maladaptation experience of children with leukaemia. To date, qualitative research on this topic available for reference has been limited. The current study had three short-term and long-term goals. The first was to explore in depth the social adaptation and problems faced by children with leukaemia of different ages through face-to-face interviews. The second was to provide a key step in developing a social adaptation assessment scale for children with leukaemia of different ages and help us refine the item pool of the Social Adaptation Rating Scale for Children with Leukemia in the future, thus contributing to the construction of a more accurate scale for evaluating the social adaptation level of children with leukaemia and achieve early prevention and timely intervention for their social adaptation problems. The last was to help improve the social adaptation of children with leukaemia so that they can return to their families and to the society without difficulty in the future.

\section{METHODS}

\section{Patient and public involvement statement}

Neither the patients nor the public were involved in the design, conduct, reporting or dissemination plans of our research.

\section{Study design}

In this qualitative study, an indepth and holistic exploration of the research phenomenon was conducted using semistructured interviews, which are especially suitable for describing research subjects' true situations and feelings. ${ }^{18}$ Data were analysed using the seven steps of Colaizzi's Method publication details method to improve the reliability of the results. ${ }^{19}$ The writing of the full text was guided by the Standards for Reporting Qualitative Research to ensure completeness and accuracy.

\section{Participants}

This study adopted a consecutive sampling strategy. According to the specific inclusion and exclusion criteria, children with leukaemia who were inpatients admitted to the department of haematology-oncology of a tertiary children's specialist hospital in Chongqing, China, from August 2020 to November 2020, were selected. Childhood is a critical period of human socialisation. The process of socialisation in children is divided according to different age stages. Eight years of age is an important age boundary. First, from the perspective of brain development, children between the ages of 8 and 10 are at the same level as adults. In addition, children aged 8 and above have a certain foundation of cultural knowledge, understanding of society and personal mental development. In addition, they have a high degree of independence and control. ${ }^{20} \mathrm{~A}_{\text {study }}{ }^{21}$ 
showed that children aged 8 years and older and teenagers have the ability to independently self-report their health and psychological feelings. For these reasons, the inclusion criteria for children with leukaemia were as follows: (1) children diagnosed with leukaemia by clinical bone marrow examination and with any disease type, including acute and chronic leukaemia, and various subtypes such as lymphocytes and myeloid leukaemia; (2) the included children were divided by age according to whether they had the ability to independently report; thus, children aged 2-18 years were divided into two groups: one group was 2-8 years old and the interviewees in this group were the primary caregivers of the children, and the other group was 8-18 years old and the children participated in the interviews themselves; (3) children had stayed in the hospital for more than 1 week; (4) children and/or their main caregivers could communicate effectively; and (5) children and their main caregivers volunteered to participate in this study and signed an informed consent form. The exclusion criteria were as follows: (1) children with mental illness or history of mental illness; and (2) children with other major diseases, such as other malignant tumours, severe cardiovascular diseases or other organic diseases. The researchers obtained general information on the children with the help of the head nurse. When selecting interview subjects, the researchers fully considered the representativeness of the research subjects and selected samples based on age, sex, disease diagnosis, number of hospitalisations, education level of the main caregiver and family's economic status. The final sample size was 20 children. There were 10 children in each age group, with 12 boys and 8 girls. The general information of the children with leukaemia is shown in table 1 .

\section{Data collection}

From August 2020 to November 2020, face-to-face semistructured interviews with the interviewees were conducted. Before the formal interview, a preliminary interview was conducted to correct and improve the interview guide and make it more fluent and practical. ${ }^{22}$ The formal interviews were conducted in a quiet and suitable place and each interview lasted approximately $30-40 \mathrm{~min}$. The interviews were conducted by the first author of this study, who first learnt interviewing skills and had a certain level of interviewing ability. The content of the revised interview outline drawn up for the different age groups is shown in table 2. Before collecting data, the researchers established a friendly and trusting relationship with the children and their main caregivers to reduce the Hawthorne effect. ${ }^{23}$ The researchers encouraged the interviewees to fully express their feelings and thoughts, while maintaining a neutral attitude during the interviews, and we objectively recorded non-verbal symbolic information such as tone, intonation and expression. At the end of the interviews, each participant received a small gift as a reward. After each interview, the data were sorted. If there were unclear responses, a second

\begin{tabular}{llr}
\hline $\begin{array}{l}\text { Table 1 } \\
\text { (N=20) }\end{array}$ & General information of children with leukaemia \\
\hline Category & Group & $\mathbf{n ~ ( \% )}$ \\
\hline Sex & Female & $8(40)$ \\
& Male & $12(60)$ \\
Age (years) & $2-7$ & $10(50)$ \\
& $8-18$ & $10(50)$ \\
Leukaemia subtype & Common ALL & $9(45)$ \\
& T-ALL & $3(15)$ \\
& B-ALL & $2(10)$ \\
Number of & AML & $6(30)$ \\
hospitalisations & $1-4$ & $11(55)$ \\
& $5-9$ & $3(15)$ \\
Main caregiver & $\geq 10$ & $6(30)$ \\
& Parents & $4(20)$ \\
& Mother & $11(55)$ \\
& Father & $4(20)$ \\
& Grandparents & $1(5)$ \\
\hline
\end{tabular}

ALL, acute lymphocytic leukaemia; AML, acute myeloid leukaemia; B-ALL, B cell acute lymphocytic leukaemia; T-ALL, T cell acute lymphocytic leukaemia.

interview was sometimes arranged with the interviewee to clarify and ensure the accuracy of the collected data. Data were collected until no new themes emerged; that is, the interviews were terminated once data saturation was achieved.

\section{Data analysis}

All analyses were conducted within 24 hours after the interview. The recording materials and the researchers' on-site observations were later transcribed, and the data were analysed using the seven steps of Colaizzi's phenomenological research and analysis: (1) read the collected information carefully and become fully familiar with it; (2) analyse the data word by word and identify and extract important and meaningful statements related to the research question; (3) construct/code the meanings of recurring and meaningful opinions; (4) collect the coded opinions, find meaningful common concepts and form the prototype of the theme; (5) describe each topic in detail and extract and add typical original statements from the interviewees; (6) put similar themes and their descriptions together for repeated comparisons, identify and extract similar views, and construct and sublimate themes; and (7) return the generated topic structure to the interviewee for verification of the content. All materials collected during the interview were entered into NVivo V.12 for qualitative data analysis. Two raters sorted and analysed the data according to the above steps. When their results were highly consistent, the reliability of the qualitative research was considered ideal, and the results were adopted to ensure the validity of the qualitative 
Table 2 Semistructured interview outline for children in different age groups

2-7 years old (interview with the main caregiver of 8-18 years old (interview with the child himself the child)

\section{or herself)}

Interview outline

1. What was your child's life like during a day in hospital? Was there any discomfort? (life and treatment)

2. Who was taking care of the child and how did the child get along with them? Could the child take care of himself or herself? How about hands-on ability?

3. Does the child have any hobbies? Did the child like 3. Do you have hobbies? Did you like something something or do something after being sick?

4. Did your child change before and after the illness? 4. Did you change before and after your illness? What were the changes? (character, behaviour, emotion, etc)

5. Does the child often experience anxiety, fear or repeated bad moods? Why? (crying, tantrums, etc)

6. Does the child have siblings and how does the child get along with them?

7. How did the child get along with strangers, and did he or she cooperate with the medical staff for treatment?

8. What help and support did the child get during hospitalisation?

9. Do you think it will be difficult for your child to return to a normal life after being discharged from the hospital? If so, please give us an example. or do something after you became sick?

1. What was your life like during a day in hospital? Was there anything you were not used to? (life and treatment)

2. Who was taking care of you and how did you get along? Could you take care of yourself? What were the changes? (character, behaviour, interpersonal communication, learning, etc)

5. Do you often experience anxiety, fear or repeated bad moods? Why? (shortness of breath, palpitations, etc)

6. Do you have siblings and how do you get along with them?

7. Did you feel that you were basically isolated from the outside world because of illness? Why?

8. What help and support did you get during your hospital stay?

9. Do you have any plans for your life after you leave the hospital? Are there any difficulties in returning to a normal life? If so, please give us an example. approach. After data analysis, the researchers confirmed the final results with the raters and each interviewee, checked whether the results were consistent with their feelings, and ensured the truthfulness and accuracy of the data. When summarising the information, all authors discussed it repeatedly until they reached consensus.

\section{Ethical considerations}

Before the interviews, the researchers introduced themselves to the children and their caregivers and informed them of the purpose and content of the study and the necessity of on-site recording. In addition, the researchers strictly followed the principle of confidentiality, replaced the names of the children with numbers and did not reveal the children's information. An informed consent form was signed by each child's main caregiver and the interviews were recorded throughout the entire process. This study completely followed the principle of voluntariness. All children had the right to withdraw at any time during the study.

\section{RESULTS}

Children with leukaemia of different ages presented different states of social adaptation and their social maladaptation experiences were not completely the same. As children with leukaemia age, various social adaptation problems can occur. Analysing the social adaptation problems and their causes at various ages is critical for targeted reduction of maladjustment in children with leukaemia and improvement of their social adaptation. The social adaptation problems of children with leukaemia of different ages are summarised in table 3.

\section{Psychological maladaptation of children with leukaemia aged} 2-7 years

This study found that with increase in hospitalisation time and in the number of hospitalisations, the level of social adaptation of children aged 2-7 years increased. Children with an outgoing personality and good family support adapted better, but most children showed obvious psychological maladjustments in the initial stage of hospitalisation, which are summarised in the following sections.

\section{Fear}

The parents of all 10 children in this age group mentioned that the children showed fear, with a frequency of mentions of 12. Fear referred to the adverse emotional reaction of a child after a long period of hospitalisation and when a series of operations were required for diagnosis and treatment. Fear was also a manifestation of the child's psychological maladjustment. According to the specific scenarios mentioned by the interviewees, the children cried because they were afraid of injections and 
Table 3 Social adaptation problems of children with leukaemia of different ages

\begin{tabular}{lll}
\hline Age & Themes & Subthemes (frequency of mentions) \\
\hline $2-7$ years old & Psychological maladjustment & Fear (12). \\
& Behavioural maladjustment & Decessive attachment (10). \\
& & Dependence on electronic products (9). \\
8-18 years old & Psychological maladjustment & Impatience and irritability (7). \\
& Behavioural maladjustment & Study interruption (7).
\end{tabular}

lumbar puncture, and their low coordination affected the treatment of the disease.

The child cried when she saw the medical staff. (N1)

The child was afraid of injections and would cry. We worry that the child will weaken and become timid in the future. (N8)

The child showed fear and was afraid of the injection at first. When hearing about the injection, he started crying and was afraid of the lumbar puncture and taking medicine. He didn't cooperate at the beginning. He was still a bit uncooperative after two months. (N9)

The child was afraid of injections. She was afraid when she arrived at the hospital and saw the nurse uniform. (N11)

\section{Excessive attachment}

Seven parents mentioned that their children had excessive attachment, with a frequency of mentions of 10 . During hospitalisation, the children experienced changes in their living environment and were forced to undergo various treatments. They were prone to insecurities. In addition, due to caregivers' love and compassion for the children, many children had strong emotional attachment and most of them showed separation anxiety. ${ }^{24}$ It was difficult for the children to experience joyful emotions, which also affected learning and development in other areas.

The child was very dependent on me (his mother) and could not leave without me. (N1)

After the child was sick, his attachment emotions were stronger, and he depended on us (parents) more. (N16)

She was very dependent on me (mother) after she was sick; I had always taken care of her. (N20)

\section{Psychological maladaptation of children with leukaemia aged 8-18 years}

This study found that there were differences in the psychological adaptation problems faced by children with leukaemia of different ages. As the children grew older, their cognitive levels continued to improve and their disease knowledge increased. Children aged 8-18 years had more psychological activities than children aged 2-7.
Generally, children with an outgoing personality, wideranging hobbies and positive family education had good social adaptation; however, some children aged 8-18 faced the following psychological adjustment problems.

\section{Impatience and irritability}

Six children mentioned being impatient and irritable, with a frequency of mentions of 7 . Drug side effects and long-term hospitalisation changed the children's temperament and they were prone to irritability and tantrums. In addition, the adolescents were in a period of psychological transition and their independence and self-awareness increased. Some children showed a rebellious psychology and were often in conflict with their caregivers, which not only affected the development of the parent-child relationship but was also not conducive to their recovery from the disease.

After I got sick, I got a bad temper. I became a little irritable and lost my temper with my mother. (N5)

After I got sick, I was very upset. If they (parents) talked too much, I would be very impatient and quarrel. Sometimes, it was annoying after hearing them talk too much, and I wanted to give up. (N17)

\section{Anxiety}

Eight children mentioned that they had anxiety, with a frequency of mentions of 10. Most children were afraid of bone puncture, lumbar puncture, chemotherapy, etc. These treatments caused them discomfort. Before each operation, the children felt nervous and anxious but showed good cooperation, which was significantly different from the younger children's expression of fear. In addition, with improvement in their cognitive abilities, older children reported that their learning and interpersonal communication needs were not satisfied due to hospitalisation and they were more worried about the adverse effects of treatment and changes to their physical appearance.

The lumbar puncture was too painful, and the bone puncture hurt more. I was afraid, but there were no other choices. I was worried that the indwelling needle would not pierce a vein so that I would need the needle to be placed many times because my blood vessels were not good for piercing. (N12) 
I was afraid of bone puncture and chemotherapy. I suffered from stomach pain during chemotherapy, but I cooperated with the doctor. (N17)

I also checked the information and learned about the disease. I was worried about recurrence. I could only take one step at a time. I had a bad interpersonal relationship in junior high school. I worried about changes in my appearance so that people who were familiar with me would look at me strangely. (N7)

\section{Behavioural maladaptation of children with leukaemia aged 2-7 years}

This study found that children with leukaemia aged 2-7 years were prone to behavioural changes due to the influence of their own disease and the surrounding environment during hospitalisation, which hindered positive development of their social behaviour. Their behavioural maladjustments are summarised in the following sections.

\section{Decrease in independent behaviour}

Seven parents mentioned that the independent behaviour of their children diminished, with a frequency of mentions of 10 . The children had a decline in physical function due to the disease and treatment. Coupled with a strong psychological attachment, they gradually lost their existing self-care ability and their independent behaviour diminished. The infant stage is a period of extremely rapid physical and functional development in children. The formation of a bad habit of overdependence could affect the development of their initiatives and independence.

The child couldn't take care of himself well. He was very dependent on me (his mother), and I had to feed him his meals. (N2)

The child was more dependent after being sick, and he could take care of himself before. (N16)

\section{Dependence on electronics}

Seven parents mentioned that their children depended on electronics, with a frequency of mentions of 9. Children between 2 and 7 years old were limited in their range of activities during hospitalisation. Communication with their peers diminished while the frequency of use of electronics rose. Many caregivers also used mobile phones to calm their children when they cried. The children relied on electronics and most of them liked watching cartoons or browsing TikTok on mobile phones.

The child preferred to spend time on the mobile phone after she was sick, and she would cry if we didn't give it to her, so we (parents) usually gave it to her. (N11)

The child was very addicted to electronics, and he would be angry if he was not allowed to play. (N16)

The child liked spending time on mobile phones after he was sick, and he often looked at his mobile phone in the ward. (N20)
Behavioural maladaptation of children with leukaemia aged 8-18 years

Adolescents with leukaemia were experiencing rapid development of intelligence and cognitive ability. Compared with early childhood, in adolescence they had more social needs and a thirst for learning. Long-term and repeated hospitalisation caused the children to face many challenges related to role conversion and environmental changes. Some children had insufficient selfregulation ability and undesirable behavioural changes were inevitable. Such changes are summarised in the following sections.

\section{Study interruption}

Six children mentioned that they had study interruptions, with a frequency of mentions of 7 . The experience of repeated hospitalisation forced the children with leukaemia aged 8-18 years to take time off or leave school and made them unable to attend school for a normal study life. Their study time was reduced and their academic performance declined. Moreover, communication and interaction with teachers and classmates were somewhat reduced and the children felt distressed and worried about the profound impacts on their future study lives.

After I got sick, I only worried about the decline in grades. (N10)

I couldn't go to school in the hospital, and I felt very uncomfortable. (N11)

I was worried about delaying my studies and repeating the grade. I was taking a leave of absence to be hospitalized. (N15)

In terms of going to school, I wanted to hang out with my classmates. I also felt that illness had delayed a lot. (N19)

\section{Addiction to electronics}

Six children mentioned that they were addicted to electronics, with a frequency of mentions of 10 . During hospitalisation, due to environmental changes and limitations, children aged 8-18 years were separated from their peers for a long time; they could not do their hobbies and their social needs could not be met. Mobile games became the main form of entertainment for these children. The long-term use of electronics and indulgence in mobile games further reduced interaction between the children and others, which was not conducive to the development of their social skills and affected their return to normal learning and life.

Because I was hospitalized, I played more games, and I would be very upset if my rank dropped. (N5)

I can talk with you while playing games; I usually play games almost all day. (N13)

I liked playing games during my hospital stay, as it was too boring. (N17) 
I like playing games with friends, and I used to play games when I was hospitalized. (N19)

\section{DISCUSSION}

Children with leukaemia of different ages have different degrees of social adaptation problems and it is critical to formulate targeted early intervention strategies

The findings of this study demonstrate that, in terms of psychological adaptation, children with leukaemia aged 2-7 years mainly had adverse emotional reactions such as fear and excessive attachment, while children aged 8-18 years mainly showed irritability, anxiety and worry, indicating that children with leukaemia of different ages experienced different psychological stresses. Han et $a l^{25}$ concluded that the feelings of children with leukaemia aged 7-14 years old during the initial period of hospitalisation were mainly sadness, fear, worry, impatience and irritability, which was consistent with the findings of this study. However, the authors of the previous study did not clarify the difference in psychological changes in children with leukaemia in childhood and adolescence. Other related studies have shown that children and adolescents with leukaemia are more likely to have symptoms such as anxiety and depression compared with their peers ${ }^{26}$ and that their suicidal tendency and absolute risk of posttraumatic stress in adulthood are higher than those of adults who have never had leukaemia, ${ }^{13}$ suggesting that the psychological adaptation problems of children with leukaemia have far-reaching effects. From hospitalisation to discharge and adulthood, children with leukaemia may be affected by negative emotions. These psychological adaptation problems need to be addressed urgently. It is essential to pay attention to children's psychological adaptation and carry out active and effective interventions.

In terms of behavioural adaptation, children with leukaemia aged 2-7 years in this study generally had the problem of reduced independent behaviour, while children aged 8-18years faced learning difficulties. There were differences in the life needs of children with leukaemia of different ages so their behavioural adaptation problems were not the same. Young children had poor autonomy and stronger emotional attachment. They hoped to be taken care of by their parents and medical staff and gain a sense of security through the close attention of caregivers. ${ }^{25}$ Older children pursued higher-level needs and began to pay attention to learning. In addition, children of any age were dependent on or addicted to electronics, but paid attention to different content, which was directly related to the increasing popularity of electronics and the fact that the children were exposed to electronic products at a younger age. A study ${ }^{27}$ showed that excessive use of electronics could lead to psychological problems such as insecurity, depression, tension and anxiety, as well as late bedtimes, impaired parent-child relationships, impaired relationships at school and various behavioural problems that are not conducive to children's physical and mental health. Other studies have shown that the overall detection rate of neurotic behavioural problems in children with leukaemia is higher than in other children of the same age ${ }^{28}$ and that survivors of childhood leukaemia are more likely to have symptoms such as antisocial behaviour and impaired social skills than their peers. ${ }^{26}$ Social problems in survivors were also evidenced by the fact that they attended school for fewer years and had a lower level of perceived social support from family, friends and significant others than the controls. ${ }^{29}$ The risk of unemployment in adulthood is also $50 \%$ higher among those who had leukaemia as a child than among the general population. ${ }^{14}$ After being ill, from short-term treatment to long-term survival, children with leukaemia exhibit various forms of behavioural maladaptation at different ages, showing a long-term and persistent trend. At present, improving children's health literacy and promoting healthy behaviours have been found to have a positive impact on enhancing children's social competitiveness in China, ${ }^{30}$ and it is increasingly important to regulate the behaviours of children with leukaemia as early as possible.

Lake and $\mathrm{Chan}^{31}$ proposed that early childhood experience affects one's life. For children with leukaemia, their lives are affected to varying degrees by experiences of psychological and physical discomfort cause by disease treatment, repeated hospitalisations leading to constant changes in their living environment, etc. When the child is not able to adjust himself or herself to adapt to changes in life, maladaptation may occur. During this time, external help is particularly important so that the child can better adapt to his or her new life and achieve mind-body balance. Therefore, during hospitalisation of children with leukaemia, it is imperative to identify and intervene in their social adaptation problems in a timely manner. According to the physical and mental characteristics of children with leukaemia of different ages, early intervention strategies can be formulated for social adaptation, which can fundamentally prevent or reduce the occurrence of social maladaptation in children with leukaemia.

\section{Analysis of the social adaptation problems of children with leukaemia of different ages and suggestions for formulating early intervention strategies \\ Psychological maladaptation \\ Fear and attachment}

According to the findings of this study, in children with leukaemia aged 2-7 years old, the main manifestations of psychological maladjustment are fear and emotional attachment. The interviewed parents reported that their children's fear mainly came from invasive operations, such as lumbar puncture and injections. Moreover, the children were afraid of just seeing the medical staff. Alleviating children's fear is an urgent problem to be solved. Qun $e t a l^{32}$ stated that the implementation of comfort care could eliminate the nervousness and fear of children with leukaemia. Regarding children's fear, medical staff should pay attention to their emotional reactions during 
hospitalisation and first ensure a comfortable and warm environment when conducting diagnosis and treatment. In addition, Xie $e t a l^{33}$ found that children with leukaemia wanted pictures of children to be hung on the wall for them to look at during lumbar puncture and for medical staff to wear overalls with colourful cartoon patterns as this could eliminate children's fear of the hospital environment and medical staff. The above research results provide a practical basis for this study to further ameliorate children's fear and promote social adaptation. Children's fear is also a manifestation of their lack of security. When children are insecure, their emotional attachments also come to the fore. Consequently, medical staff should pay attention to displaying kindness, establishing a trusting relationship with children, increasing children's sense of security and improving emotional attachment.

\section{Impatience, irritability and anxiety}

First, due to adverse reactions to disease treatment and the limitations of the hospital environment, children are prone to impatience, irritability and other adverse emotions. Effectively alleviating these negative emotions will help children not only establish harmonious interpersonal relationships but also recover from the disease. For example, sand play therapy is a kind of psychotherapy suitable for children with emotional and behavioural disorders $^{34}$ and can be considered for children with leukaemia. This type of therapy helps children express themselves and speak frankly from their hearts in a safe environment, clarify and change distorted and unhealthy modes of thinking, and liberate themselves from negative and repressed emotions. In addition, because of their lack of disease-related knowledge, children feel anxious about the prognosis of the disease. Medical staff should give timely explanations and encourage children to adapt to the series of changes brought about by the disease and to better cooperate with the treatment. During hospitalisation, through resilience interventions for stress management,${ }^{35}$ children's coping ability and appropriate use of relaxation techniques can be improved, such as taking deep breaths to relieve tension and anxiety. At the same time, to effectively alleviate children's anxiety, medical staff and parents should strengthen communication with children, encourage children to express their ideas, always understand their needs and help them solve problems as much as possible.

\section{Behavioural maladaptation}

\section{Decrease in independent behaviour}

The ages of 2-7 years represent a critical period of development in children's social behaviour, including social communication and life abilities. The results of this study found that children aged 2-7 were prone to reduced independent behaviour, which meant that they were unwilling to do what they had the ability to do after illness and were more dependent on their caregivers. Such behaviour was not conducive to their operational ability and intellectual development. On the one hand, children have insufficient energy due to the impact of their disease; on the other hand, the caregivers worried about the children's physical health and took care of all matters. Young children still have insufficient understanding and therefore parents or caregivers should adjust their own mentalities and provide children with correct education and guidance during their normal development stage. Objectively, children need careful care, but caregivers should not be overprotective to avoid depriving children of the opportunity to learn independently and hindering the development of their hands-on ability. Consequently, caregivers should reconsider the approach of taking care of all of the children's needs and instead should maintain children's self-care ability as much as possible within their ability, encourage children at appropriate times, and praise positive and correct behaviours to enhance children's self-confidence. Moreover, having multiple caregivers alternate in providing care is recommended to avoid overdependence on one caregiver.

\section{Learning interruption}

The results of this study suggest that learning interruption occurs in children with leukaemia aged 8-18 years. The learning needs of children at these ages must be met to reduce the effect of the disease on their academic development. Given the vigorous development of online education during the COVID-19 epidemic, children with leukaemia could use online resources to learn independently. In addition, during hospitalisation, it could be arranged for children of similar ages to live on the same ward when conditions allow, and a relatively quiet ward environment with a better learning atmosphere could be provided as much as possible to alleviate the difficulty that children face in not being able to study normally due to hospitalisation.

\section{Reliance on or addiction to electronics}

Children with leukaemia of all ages had problems using electronics for long periods during hospitalisation. This not only affected their vision but was also not conducive to their normal rest. Controlling children's use of electronics has far-reaching significance. On the personal side, young children should play more games and play with toys instead of electronics. In addition, older children should be encouraged to develop more hobbies and social skills and strengthen communication with others. On the family side, caregivers need to impose strict requirements and supervise the children at all times, adopt a positive and optimistic family parenting method, ${ }^{36}$ accompany children in exposing them to unfamiliar aspects of the world, and make video games only one of their many interests. In terms of social support, it is recommended that leisure activity areas be created on the ward. During the period of diagnosis and treatment, for children's safety, the number of leisure activities and the types of entertainment for children should be increased and communication and interaction between peers should be strengthened to effectively prevent children 
from indulging in independent use of electronics for long periods of time. In addition, with the popularisation of smartphones, some medical institutions have promoted the cognitive, physical and mental health of children and adolescents through virtual 'toys' based on digital media, ${ }^{37}$ and electronic games have been initially used to support children's health and health management. ${ }^{38}$ In the future, we can design scientific and practical health management game software or game-based social adaptation assessment tools for children with leukaemia so that they can correctly evaluate and improve their physical and mental health through games. At the same time, based on scientific evidence, it is helpful for children to use electronics in a rational manner.

\section{CONCLUSION}

In this study, 20 Chinese children with leukaemia or their caregivers were interviewed using qualitative research methods and the adverse experiences of social adaptation of children with leukaemia of different ages were discussed. This paper summarised the psychological and behavioural adaptation problems they faced and proposed targeted early intervention strategies, providing a reference basis for clinically promoting the social adaptation of children with leukaemia.

\section{RELEVANCE TO CLINICAL PRACTICE}

In the future, to reduce the social adaptation problems of children with leukaemia and ultimately improve their quality of life in the long term, we could strengthen the research on early identification and intervention for social maladaptation of children with leukaemia. First, we could compile a set of social adaptation assessment scales with good reliability and validity that are applicable to children with leukaemia of different ages. Next, we could apply the scale in the clinic to investigate the status quo of the social adaptation of children with leukaemia and explore in depth the key influencing factors, further constructing a risk prediction model for social adaptation in children with leukaemia. Finally, we could construct early warning and intervention strategies to alleviate the risk of social maladaptation during hospitalisation to solve the problem of social maladaptation in children with leukaemia in a timely manner.

\section{Author affiliations}

${ }^{1}$ School of Pediatrics, Chongqing Medical University Affiliated Children's Hospital, Chongqing, China

${ }^{2}$ National Clinical Research Center for Child Health and Disorders, Chongqing, China ${ }^{3}$ Ministry of Education Key Laboratory of Child Development and Disorders, Chongqing, China

${ }^{4}$ Chongqing Key Laboratory of Paediatrics, Chongqing, China

${ }^{5}$ Nursing Department, Chongqing Medical University Affiliated Children's Hospital, Chongqing, China

${ }^{6}$ Department of VIP Outpatient, Chongqing Medical University Affiliated Children's Hospital, Chongqing, China

${ }^{7}$ Department of Haematology Oncology, Chongqing Medical University Affiliated Children's Hospital, Chongqing, China
${ }^{8}$ Research Centre of Brain and Cognitive Neuroscience, Liaoning Normal University, Dalian, China

${ }^{9}$ Key Laboratory of Brain and Cognitive Neuroscience, Dalian, Liaoning, China

Acknowledgements We would like to thank the patients who were interviewed for this study and the strong support from the head nurse.

Contributors XT wrote the main manuscript, and QL and YiL prepared the semistructured interview outline. $L Y$ and $Y L$ provided help and support in collecting the data. CG provided methodological guidance. LM checked the manuscript as the guarantor. All authors reviewed the manuscript.

Funding Funding was provided by Chongqing Science and Technology Commission (cstc2018jscxmsybX0110).

Competing interests None declared.

Patient and public involvement Patients and/or the public were not involved in the design, or conduct, or reporting, or dissemination plans of this research.

Patient consent for publication Not required.

Ethics approval This study involves human participants and was approved by the Ethics Committee of Children's Hospital of Chongqing Medical University (batch number (2020) ethical review (research) no 5). Participants gave informed consent to participate in the study before taking part.

Provenance and peer review Not commissioned; externally peer reviewed.

Data availability statement Data are available upon reasonable request. The data presented in this study are available from the corresponding author on request.

Open access This is an open access article distributed in accordance with the Creative Commons Attribution Non Commercial (CC BY-NC 4.0) license, which permits others to distribute, remix, adapt, build upon this work non-commercially, and license their derivative works on different terms, provided the original work is properly cited, appropriate credit is given, any changes made indicated, and the use is non-commercial. See: http://creativecommons.org/licenses/by-nc/4.0/.

ORCID iD

Xinyue Tang http://orcid.org/0000-0002-7624-1148

\section{REFERENCES}

1 Zhang J, Yan L, Qiu H, et al. Social adaptation of Chinese left-behind children: systematic review and meta-analysis. Child Youth Serv Rev 2018;95:308-15.

2 Tremolada M, Taverna L, Bonichini S, et al. The developmental pathways of preschool children with acute lymphoblastic leukemia: communicative and social sequelae one year after treatment. Children 2019;6:92.

3 Okado Y, Rowley C, Schepers SA, et al. Profiles of adjustment in pediatric cancer survivors and their prediction by earlier psychosocial factors. J Pediatr Psychol 2018;43:1047-58.

4 Yu L, Mo L, Tang Y, et al. Effects of nursing intervention models on social adaption capability development in preschool children with malignant tumors: a randomized control trial. Psychooncology 2014;23:708-12.

5 Tremolada M, Taverna L, Bonichini S, et al. Pediatric patients treated for leukemia back to school: a Mixed-Method analysis of narratives about daily life and illness experience. Behav Sci 2020;10:107-21.

6 Bahoush G, Nojoomi M. Frequency of cytogenetic findings and its effect on the outcome of pediatric acute lymphoblastic leukemia. Med Arch 2019;73:311-5.

7 Chinanews.com, Shanghai. The incidence, diagnosis and treatment of childhood leukemia in China: the registration rate of most new cases of acute lymphoblastic leukemia is 42.9 per million [EB/OL]. Available: Http://www.sh.chinanews.com/yljk/2020-0725/79010. shtml,2020-7-25.China

8 The Lancet Haematology. Childhood cancer on the agenda. Lancet Haematol 2019;6:e285.

9 Maloney KW, Devidas M, Wang C, et al. Outcome in children with standard-risk B-cell acute lymphoblastic leukemia: results of children's Oncology Group trial AALL0331. J Clin Oncol 2020;38:602-12.

10 GBD 2017 Childhood Cancer Collaborators. The global burden of childhood and adolescent cancer in 2017: an analysis of the global burden of disease study 2017. Lancet Oncol 2019;20:1211-25.

11 D'Agostino NM, Edelstein K, Zhang N, et al. Comorbid symptoms of emotional distress in adult survivors of childhood cancer. Cancer 2016;122:3215-24. 
12 Anestin AS, Lippé S, Robaey P, et al. Psychological risk in longterm survivors of childhood acute lymphoblastic leukemia and its association with functional health status: a PETALE cohort study. Pediatr Blood Cancer 2018;65:e27356.

13 Gunnes MW, Lie RT, Bjørge T, et al. Suicide and violent deaths in survivors of cancer in childhood, adolescence and young adulthood-A national cohort study. Int J Cancer 2017;140:575-80.

14 Mader L, Michel G, Roser K. Unemployment following childhood cancer. Dtsch Arztebl Int 2017;114:805-12.

15 Ford JS, Kawashima T, Whitton J, et al. Psychosexual functioning among adult female survivors of childhood cancer: a report from the childhood cancer Survivor study. J Clin Oncol 2014;32:3126-36.

16 Ritenour CWM, Seidel KD, Leisenring W, et al. Erectile dysfunction in male survivors of childhood cancer-a report from the childhood cancer Survivor study. J Sex Med 2016;13:945-54.

17 Choo CC, Chew PKH, Tan P, et al. Health-Related quality of life in pediatric patients with leukemia in Singapore: a cross-sectional pilot study. Int J Environ Res Public Health 2019;16:2069.

18 van Manen M. Phenomenology in its original sense. Qual Health Res 2017;27:810-25.

19 Yuan M-M, Peng X, Zeng T-Y, et al. The illness experience for people with amyotrophic lateral sclerosis: a qualitative study. J Clin Nurs 2021:30:1455-63.

20 Chen X. Discussion on the rationality of the revision of the minimum age limit of minors' limited capacity for civil conduct in the General Provisions of the Civil Law. Legal System Expo 2019:235.

21 Irwin DE, Varni JW, Yeatts K, et al. Cognitive interviewing methodology in the development of a pediatric item bank: a patient reported outcomes measurement information system (PROMIS) study. Health Qual Life Outcomes 2009;7:3.

22 Yujun J, Jing Z. Content analysis of interview outline design method in phenomenology research method of nursing thesis. Journal of Nursing of the people's Liberation Army 2016.

23 Berthelot J-M, Nizard J, Maugars Y. The negative Hawthorne effect: explaining pain overexpression. Joint Bone Spine 2019;86:445-9.

24 Barone R, Gulisano M, Cannata E, et al. Self- and Parent-Reported psychological symptoms in young cancer survivors and control Peers: results from a clinical center. J Clin Med 2020;9:3444.

25 Han J, Liu J-E, Xiao Q, et al. The experiences and feelings of Chinese children living with leukemia: a qualitative study. Cancer Nurs 2011;34:134-41.

26 Brinkman TM, Recklitis CJ, Michel G, et al. Psychological symptoms, social outcomes, socioeconomic attainment, and health behaviors among survivors of childhood cancer: current state of the literature. $J$ Clin Oncol 2018;36:2190-7.

27 Sahu M, Gandhi S, Sharma MK. Mobile phone addiction among children and adolescents: a systematic review. J Addict Nurs 2019;30:261-8

28 Lin M, Yan T, Xiaoyan H. Study on behavioral problems and influencing factors in children with malignant tumor in different age stages. Journal of Chongqing Medical University 2013;38:105-8.

29 Tremolada M, Bonichini S, Basso G, et al. Perceived social support and health-related quality of life in AYA cancer survivors and controls. Psychooncology 2016;25:1408-17.

30 Guo S, Naccarella L, Yu X, et al. Health literacy and its mediating role in predicting health behaviors among Chinese secondary students. Asia Pac J Public Health 2021;33:76-83.

31 Lake A, Chan M. Putting science into practice for early child development. Lancet 2015;385:1816-7.

32 Qun L, Ying D, Yang Li etc. Effect of comfort nursing in lumbar puncture and intrathecal injection in children with leukemia. Nursing practice and Research 2013;10:47-8.

33 Xie A, Shan Y, Niu ME, et al. Experience and nursing needs of school-age children undergoing lumbar puncture during the treatment of acute lymphoblastic leukaemia: a descriptive and qualitative study. J Clin Nurs 2017;26:3328-35.

34 Judan T, Huaying Y, Li L. Application of sand table play therapy in the treatment of psychological and behavioral problems in children with chronic diseases[J]. China Maternal and Child Health 2020;35:4638-46.

35 Steineck A, Bradford MC, Lau N, et al. A Psychosocial Intervention's Impact on Quality of Life in AYAs with Cancer: A Post Hoc Analysis from the Promoting Resilience in Stress Management (PRISM) Randomized Controlled Trial. Children 2019;6:124.

36 Competitive recruitment. Study on the relationship between selfreported health outcome and family management style in children with leukemia [D. Henan: Henan University, 2017.

37 Healey A, Mendelsohn A, COUNCIL ON EARLY CHILDHOOD. Selecting appropriate toys for young children in the digital era. Pediatrics 2019;143:e20183348.

38 Peñuelas-Calvo I, Jiang-Lin LK, Girela-Serrano B, et al. Video games for the assessment and treatment of attention-deficit/hyperactivity disorder: a systematic review. Eur Child Adolesc Psychiatry 2022;31:5-20. 